\title{
CONTEXTO DE LA EDUCACIÓN EN HONDURAS
}

Nelson Raudales García, Universidad Nacional Autónoma de Honduras, Instituto de Investigaciones Económicas y Sociales (IIESUNAH), Ciudad universitaria edifico c2 primer piso. Tel./Fax: 504-22348973

Correo electrónico: nraudales@iies-unah.org

\section{RESUMEN}

La educación constituye el medio para lograr el desarrollo de vida de la población, la que permite migrar socialmente de un estadio a otro. Asimismo la adquisición de mejores niveles educativos contribuye al desarrollo comunitario ${ }_{2}$ al poner en éste la transferencia de conocimientos aplicativos con el fin de lograr trascender a nivel regional y nacional y por ende al mejoramiento a nivel global de la economía del país.

Sin embargo, a pesar de existir grandes avances en cuanto la aplicación de políticas educativas para el mejoramiento de los niveles escolásticos de la población, aún persisten indicadores muy bajos, para el año 2010 se observa un analfabetismo alrededor del $15 \%$, además a nivel rural dicha tasa oscila en un $22 \%$ mientras que en el área urbana apenas llega al 8\% aproximadamente. Cuando se desagrega dicho indicador por edades es preocupante describir que hasta los 24 años de edad dicha tasa llega al 10\% (INE, 2010). Cuando se observan y describen las tasas de asistencia escolar a nivel longitudinal, es preocupante considerar que a partir de la década del 1990 hasta 2010 dichas tasas siguen siendo aun bajas para el periodo en mención.

Palabras clave: Educación superior, Oferta y Demanda. 


\section{CONTEXT OF EDUCATION INHONDURAS}

Nelson Raudales, Universidad Nacional Autónoma de Honduras, Instituto de Investigaciones Económicas y Sociales (IIESUNAH), Ciudad universitaria edifico c2 primer piso. Tel./Fax: 504-22348973

Correo electrónico: nraudales@iies-unah.org

\section{E\&A}

\section{IIES}

\section{ABSTRACT}

Education is the means to develop people's lives, which can migrate from one social stage to another. Also the acquisition of better levels of education contributes to community in

development by putting in this knowledge transfer applications to achieve transcend regional and national level and thus to improve the global economy.

However, although there is great progress in the implementation of educational policies to improve scholastic standards of the population, there are still very low indicators, for 2010 there is an illiteracy about 15\%, also in rural this rate varies by $22 \%$ while in urban areas is barely about $8 \%$. When this indicator is disaggregated by age is worrisome describe even the 24 years this rate reaches $10 \%$ (INE, 2010). When observe and described the attendance rates at length, it is disturbing to consider that from the decade of 1990 to 2010 these rates are still low even for the period in question.

Keywords: Higher education, Supply and demand. 


\section{INTRODUCCIÓN}

El presente artículo comprende elementos que se han considerado como insumos necesarios para el proyecto de investigación denominado Oferta y Demanda: Cadena de Valor y Suministro integrado de la educación superior ${ }^{1}$. En tal sentido, dicho informe enuncia en su apartado (1) los objetivos del presente trabajo, el apartado (2) menciona las principales fuentes de información utilizadas para enumerar los indicadores que se enuncian en el trabajo, el apartado (3) menciona la metodología del desarrollo del trabajo, la cual consiste básicamente en análisis descriptivo de indicadores recolectados de la fuentes de información que editan y sistematizan los datos, el apartado (4) describe de manera resumida algunos antecedentes de la educación en honduras, el apartado (5) describe el funcionamiento del sistema educativo Hondureño, los sub apartados (6.1 al 7.2) describen algunos resultados encontrados del proceso de abstracción de los indicadores identificados para tal fin, el apartado (8) enumera algunas consideraciones finales de dicho trabajo y finalmente se describe la principal bibliografía utilizada durante la descripción y análisis correspondiente.

\section{OBJETIVO DEL TRABAJO}

El objetivo del presente documento es dar a conocer al público lector, sobre las variaciones de los indicadores educativos del nivel primario, secundario y educación superior en Honduras. Se analizan dichos insumos a nivel de género, desagregaciones urbano-rurales, etc.

\section{FUENTES DE INFORMACIÓN}

Las principales fuentes de datos utilizadas para elaborar y construir los indicadores que se describen a continuación surgen del acceso a fuentes secundarias de información que se encargan de recolectar, sistematizar, organizar y construir los insumos educativos previamente descritos, entre dichas instituciones se puede mencionar: La Dirección de Educación Superior (DES) de la UNAH, Secretaría de Educación de Honduras (SEH), e Instituto Nacional de Estadísticas (INE).

\section{METODOLOGÍA}

La metodología para el desarrollo del presente documento es de naturaleza descriptiva y consiste en la recolección, organización, síntesis y análisis de los 
insumo para el estudio de Oferta y Demanda; para ello se inicia con la identificación de las instituciones proveedoras de datos necesarias para la recopilación de indicadores longitudinales observados en las diversas fuentes de datos mencionadas previamente.

\section{FUNCIONAMIENTO DEL SISTEMA EDUCATIVO HONDUREÑO}

De acuerdo a la Constitución de la República de Honduras, la educación es función esencial del Estado para la conservación, el fomento y difusión de la cultura, la cual deberá proyectar sus beneficios a la sociedad sin discriminación de ninguna naturaleza. En tal sentido, los niveles educativos están organizados por niveles que corresponden a: educación pre básica, educación básica, educación media y por último la educación superior.

En el nivel de educación pre básica comprende los centros de educación maternales, pre kínder, jardines de niños y los centros pre básicos.

En el nivel de educación básica, se sub divide en tres niveles integrados por mismo número de ciclos donde se encuentra el nivel de educación media.

Finalmente, en el caso de la Educación Superior la Constitución de la Republica de Honduras le otorga a la UNAH las funciones de rectorar, coordinar y supervisar dicho nivel, así como los CES encargados de ofrecer la oferta académica correspondiente.

\section{RESULTADOS}

A continuación se describe el comportamiento de las variables educativas y se realiza un breve análisis descriptivo de los indicadores educativos, cabe mencionar que los mismos corresponden a insumos del nivel de educación primaria, media y superior, ello considerándolos como elementos de entrada y de procesos en la cadena de suministro de la educación superior.

\subsection{Indicador de analfabetismo por dominio}

De acuerdo a la Encuesta de Hogares de Propósitos Múltiples (EPHPM) del Instituto Nacional de Estadísticas de Honduras (INE; 2010), el analfabetismo es un problema muy importante en la realidad educativa del país. De acuerdo a la misma fuente para dicho año el $15.2 \%$ de las personas mayores de 15 años no saben leer ni escribir, los datos indican que la tasa de analfabetismo continúa siendo mayor en la población del área rural con un $22.3 \%$ frente a un $7.8 \%$ en el área urbana (Gráfico No.1).

Asimismo, es considerable mencionar que las personas en esta edad pueden tener dificultad para obtener un empleo e insertarse al mercado laboral y contribuir de dicha forma en aportar a la economía del país. Además a nivel de dominios, la tasa de analfabetismo se mantiene considerablemente alta, ello se 
observa reflejado más a nivel rural en comparación con el área urbana.

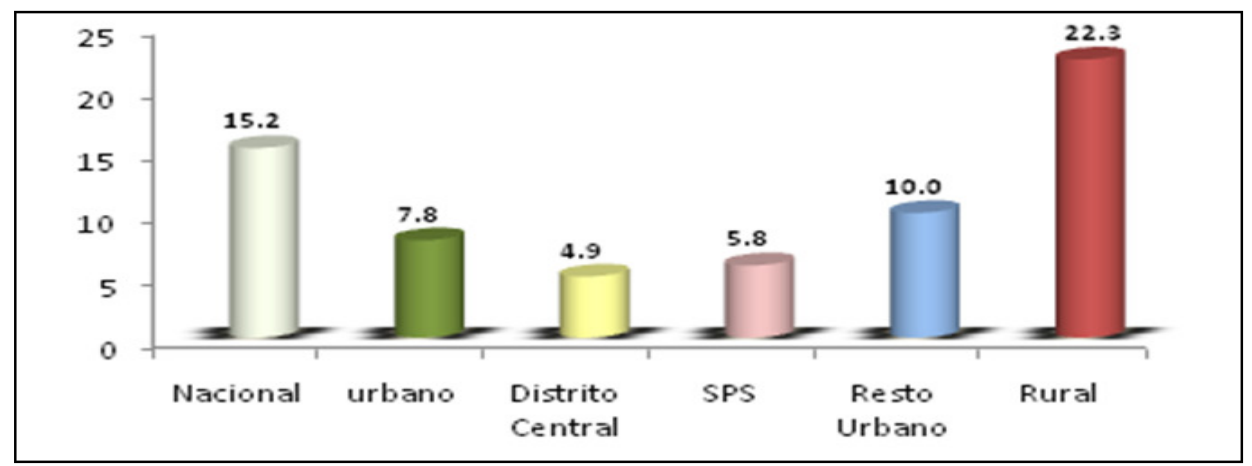

Gráfico 1, Honduras: Tasas de Analfabetismo según dominio, 2010 (Fuente: Elaboración propia en base a INE, EPHPM, Mayo 2010)

\subsection{Analfabetismo por edades}

Al considerar el analfabetismo por edades éste es superior en las personas de mayor edad, sin embargo, hasta los 24 años la tasa se ubica en 10\%, a partir de los 30 años dicha cifra aumenta hasta alcanzar su máximo en la población de 60 años y más, de la cual casi la mitad (43.3\%) no sabe leer ni escribir (Gráfico No. 2).

Además este nivel de analfabetismo en las edades antes mencionadas y las edades posteriores se vuelve un indicador de suma importancia, particularmente por la carga económica que dichos jóvenes pueden representar para sus padres, asimismo tal situación se vuelve limitante particular para que esta población se inserte en la actividad productiva del país.

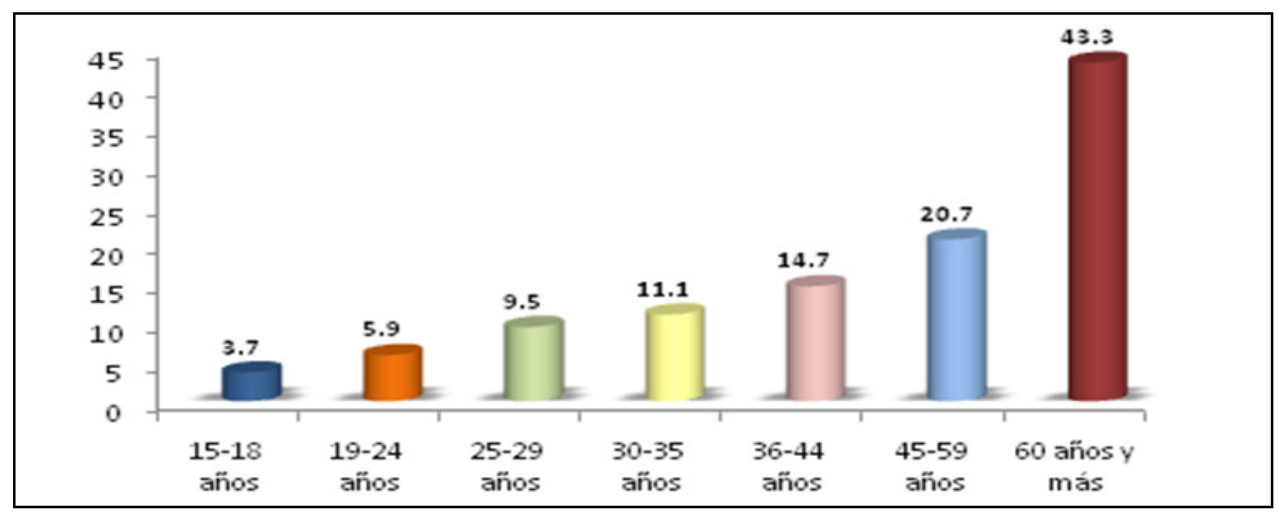

Gráfico 2, Honduras: tasa de analfabetismo según edades, 2010

(Fuente: Elaboración propia en base a INE, EPHPM, Mayo 2010) 


\subsection{Analfabetismo a nivel nacional}

Dicho indicador puede considerarse como una de las principales limitantes, debido a que puede llegar a impedir el desarrollo de los países. Lógicamente existen evidencias por medio de algunas medidas estadísticas que demuestran altos niveles de asociación entre la vida decorosa de las personas y los altos niveles educativos y por ende el nivel de desarrollo científico y tecnológico de los países. Además este indicador puede considerarse en una de las principales barreras para que los ciudadanos de un país, un estado y de un pueblo puedan desarrollar niveles de vida adecuados, desarrollar mejores niveles de cohesión social, etc. Sin embargo, al observar dicho indicador se refleja que éste ha descendido durante el periodo observado, no obstante aún se mantiene alto alrededor del 16\% (Gráfico No.3)

E\&A IIES

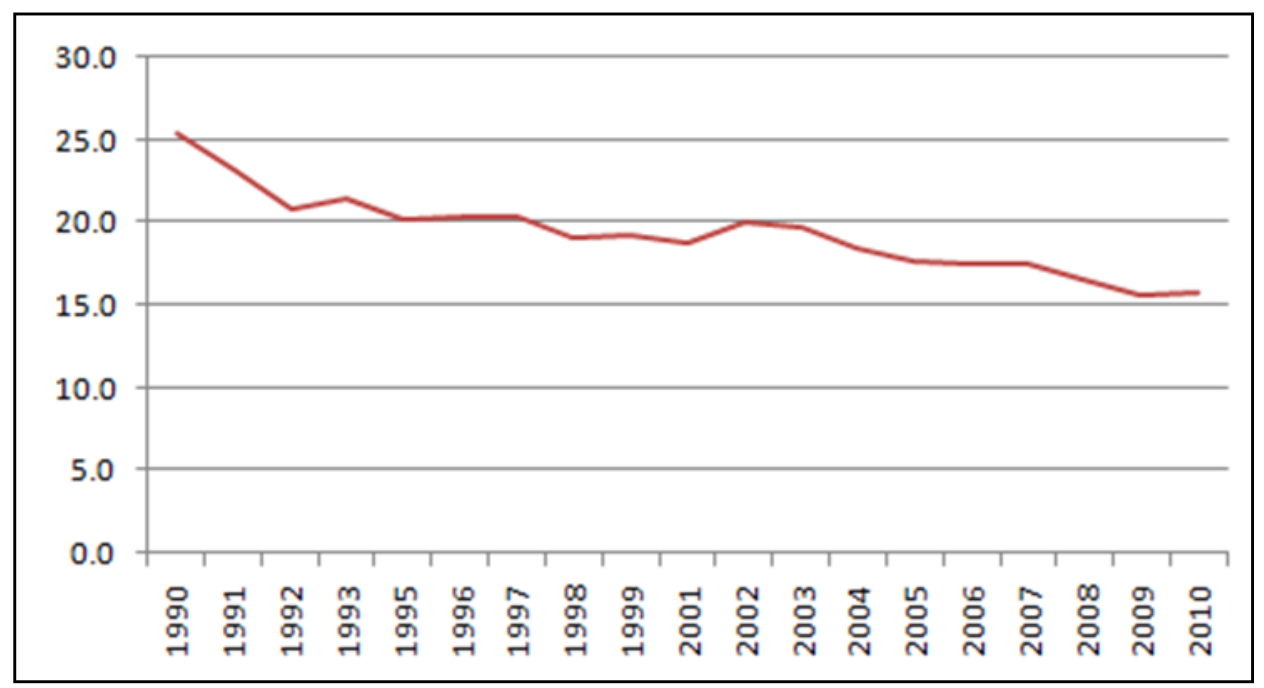

Gráfico 3, Honduras: tasa de analfabetismo total, 1990-2010.

(Fuente: Flores, M. 2011)

\subsection{Deserción escolar}

La deserción escolar es otro indicador a nivel educativo el cual se debe brindar la debida importancia por parte de las autoridades pertinentes, ello debido a que el Estado invierte cantidades considerables en tratar de educar a la población en los niveles correspondientes, y bajo esas circunstancias se debe buscar los medios para evitar que los educandos indistinto el nivel en el que se encuentren, abandonen sus estudios (Gráfico No. 4) Sin embargo, las cifras indican que a nivel de país los educandos abandonan las aulas educativas en donde reciben el pan del saber por múltiples razones las cuales pueden requerir mayor nivel de estudio; para éste indicador se reporta un $0.8 \%$ de deserción a nivel nacional, siendo ésta en el área rural de $0.9 \%$ y 0.7 para el área urbana (INE, 2010). 


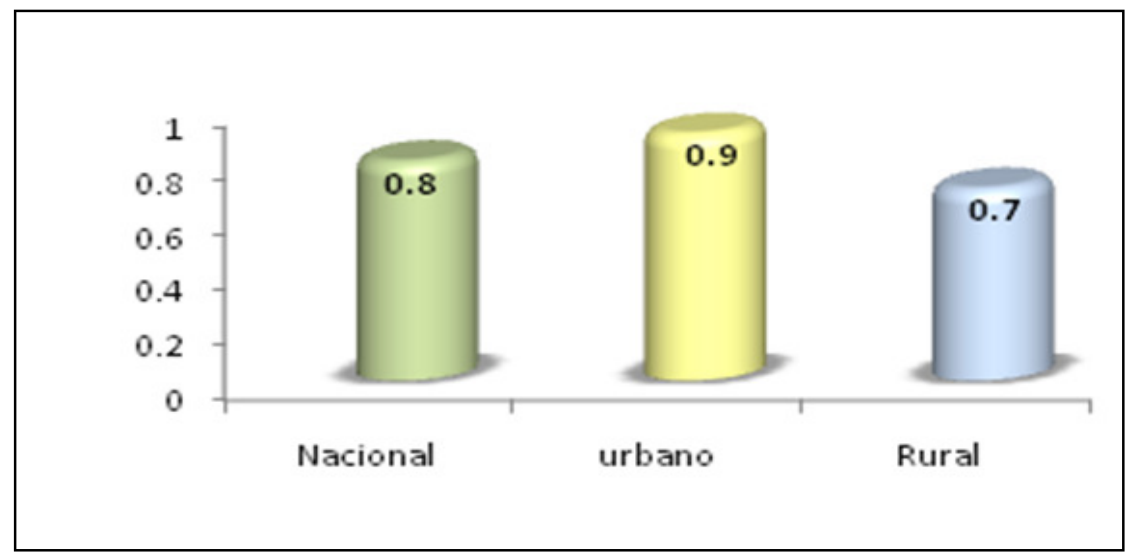

Gráfico 4, Honduras: tasa de deserción escolar según área de residencia, 2010. (Fuente: Elaboración propia en base a INE, EPHPM, Mayo 2010)

\subsection{Asistencia escolar}

Si bien es cierto, a partir de los indicadores descritos previamente, se ha reflejado que ha existido índices no tan halagadores, también es viable mencionar que a nivel de la asistencia a centros escolares en el nivel que corresponde, los mismos mantienen tendencias con variaciones relativamente bajas (gráfico No. $5)$.

Sin embargo, ello no es obstáculo para describir que el nivel de educación primaria es el que tiene mayores índices de asistencia, es decir, del total de los educandos que deberían estar asistiendo a los diversos niveles educativos logran permanecer 9 de cada 10 estudiantes del nivel de primaria, no así para los siguientes niveles (ciclo común, diversificado y el nivel de pre escolar) quienes reflejan tasas mucho más bajas (INE, 2010). Cabe mencionar que deberían realizarse esfuerzos conjuntos entre los diversos actores del sistema educativo (padres de familia, maestros, educandos, gobierno, asociaciones de cooperación, etc.) para permitir el desarrollo y la aplicación de políticas públicas orientadas a lograr mejores niveles de asistencia escolar en los niveles que muestran tasas sumamente bajas (diversificado, ciclo común). 


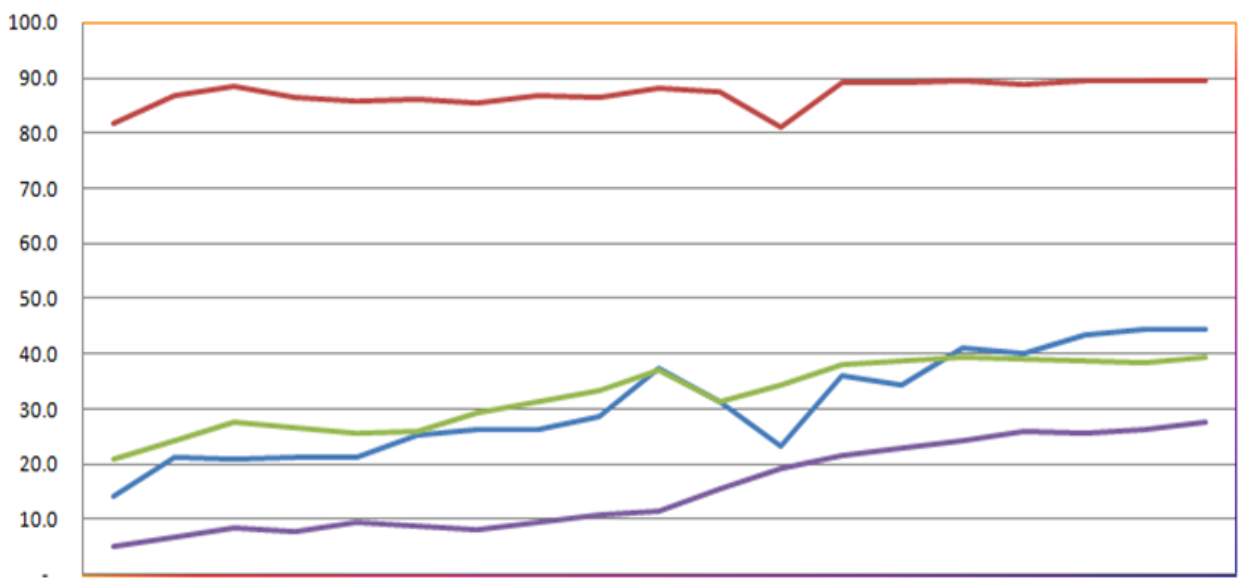

$\begin{array}{lllllllllllllllllll}1990 & 1991 & 1992 & 1993 & 1995 & 1996 & 1997 & 1998 & 1999 & 2001 & 2002 & 2003 & 2004 & 2005 & 2006 & 2007 & 2008 & 2009 & 2010\end{array}$

- Pre-Escolar - Primaria Ciclocomun Diversificado

Gráfico 5, Honduras: tasa de asistencia según niveles primaria y media, 2010. (Fuente: Flores, M. 2011.)

\subsection{Situación de la educación superior en Honduras ${ }^{3}$}

E\&A IIES

La educación superior Hondureña data desde el año de 1847, transcurriendo 130 años con solamente una institución de educación de ese nivel, no es sino hasta 1978 cuando se abren dos nuevos centros, en la década de los ochenta cuatro centros (UNAH, 1985), en la década de los noventa seis centros y en la última década siete centros para hacer un total de veinte centros (DES, 2008). En este devenir del tiempo se ha pasado de grandes campus urbanos a multicampus que aunque también son urbanos están diseminados en los principales centros urbanos del país.

La oferta educativa de educación superior actual es de 349 grados, de los cuales la mitad de esta es ofrecida por instituciones públicas. El 55\% de la oferta de grados es ofrecida a nivel de licenciatura, siendo el grado académico de mayor oferta y aceptación estudiantil para realizar estudios, aun cuando no hay que olvidar que los grados superiores a las licenciaturas fueron ofertados más recientemente (DES, 2008).

A través del tiempo ha existido una concentración geográfica de la educación superior, primero en la ciudad capital y desde la década del sesenta se ubicó en las tres principales ciudades del país (considerados como polos de desarro1lo), a inicios de la década de los ochenta se expandió a otras ciudades del occidente, sur, centro y oriente del país, primero en la oferta de centros de naturaleza pública y más recientemente de carácter privado, principalmente desde 
fines de los noventa e inicios del dos mil. Aun así, persiste una alta concentración educativa en las dos principales ciudades del país, que son Tegucigalpa y San Pedro Sula.

La matricula en la educación superior púbica es mayoritaria con una tendencia a la disminución, en la última década disminuye su proporción del $86 \%$ al $64 \%$ (DES, 2008). En el caso de los matriculados de primer ingreso ha pasado de $81 \%$ al $52 \%$ y en el año 2007 por primera vez la matrícula de educación privada superó a la pública como un hito histórico.

En las áreas del conocimiento, hay una alta concentración de la oferta educativa en el sector de ciencias sociales, ciencias comerciales y derecho, que sumadas a la enseñanza de la educación y humanidades alcanza el 57\% (DES, 2008). Las ciencias y las ingenierías alcanzan solamente el $21 \%$, la salud el $12 \%$ y el resto agricultura y los servicios.

La cobertura educativa de la educación superior en el país es baja, alcanza apenas el $15 \%$ y su avance a través del tiempo ha sido lento, desde el año de 1997 que era de $7 \%$, transcurrieron 18 años para duplicarse y alcanzar el nivel actual. En relación a la modalidad de estudios, la educación presencial ha reducido su participación del total en un diez por ciento, actualmente es de $78 \%$, la educación a distancia es $22 \%$ y la virtual es insignificante, aun así persiste la educación tradicional presencial como modalidad educativa mayoritaria.

Los graduados de educación superior en la última década han representado entre el 6 al 10\% del total de matriculados y del 2 al $6 \%$ de los matriculados de primer ingreso, tendencias que han ido creciendo a lo largo del tiempo, e incluso los graduados tienen tasas superiores de crecimiento en comparación con las de matrícula.

\subsection{Matricula de la educación superior}

La educación superior hondureña en los última década ha alcanzado los niveles más grandes de su historia, es los últimos trece años se incrementó el número de estudiantes matriculados en tres veces, al 2008 el número de efectivos supera los ciento cincuenta mil estudiantes (Gráfico No.6).

Por modalidad en el país la educación presencial es la preponderante, ya que la modalidad virtual ha sido insignificante e incluso ha desaparecido del sistema, mientras que la educación a distancia en el periodo estudiado aumentó su número y proporción, sin embargo, apenas supera un cuarto del total.

En la matricula total de educación superior se ha producido un cambio significativo en el volumen, aunque los centros de educación superior pública contribuyen con los mayores volúmenes, en los últimos dos años ha disminuido su 


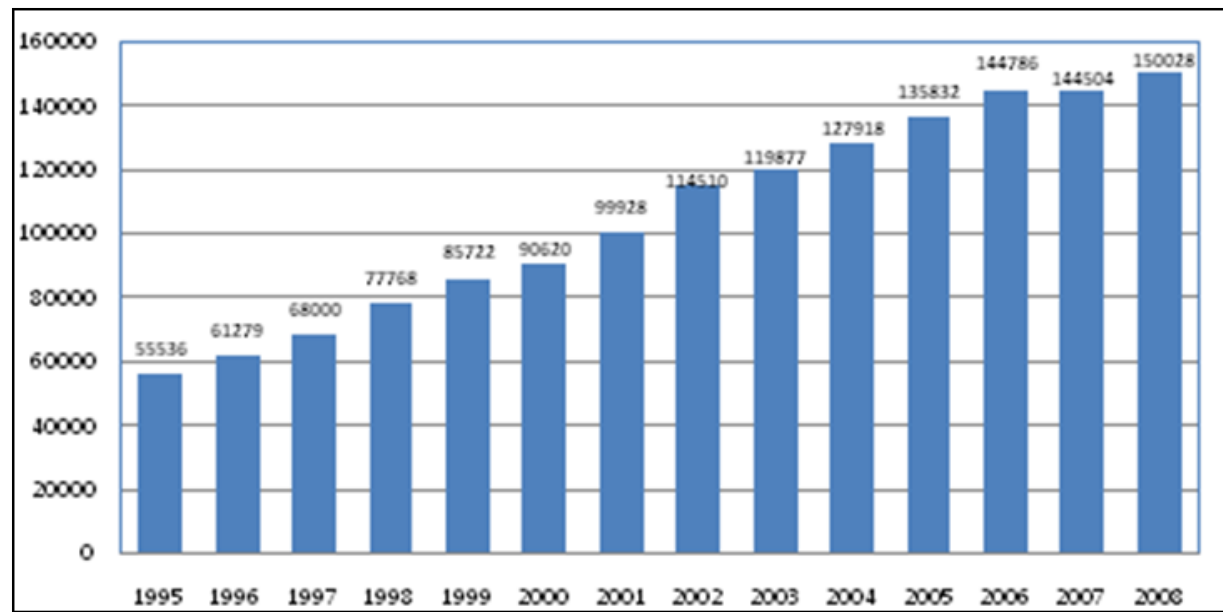

Gráfico 6, Honduras: Matrícula total en la Educación Superior, 1995-2008 (Fuente: Flores, M. 2011.)

número, básicamente por la reducción en la matrícula de la Universidad Nacional Autónoma de Honduras (UNAH), principal contribuyente de la educación superior en el país, que inclusive generó tasas de crecimiento interanual negativas y desde luego, por el incremento de matrícula en los centro de educación superior privada. En los últimos años la proporción de matriculados de

E\&A IIES educación superior privada se ha incrementado, de $13 \%$ a $35 \%$, la cual también es una tendencia general a nivel la región latinoamericana. (Gráfico No. 7)

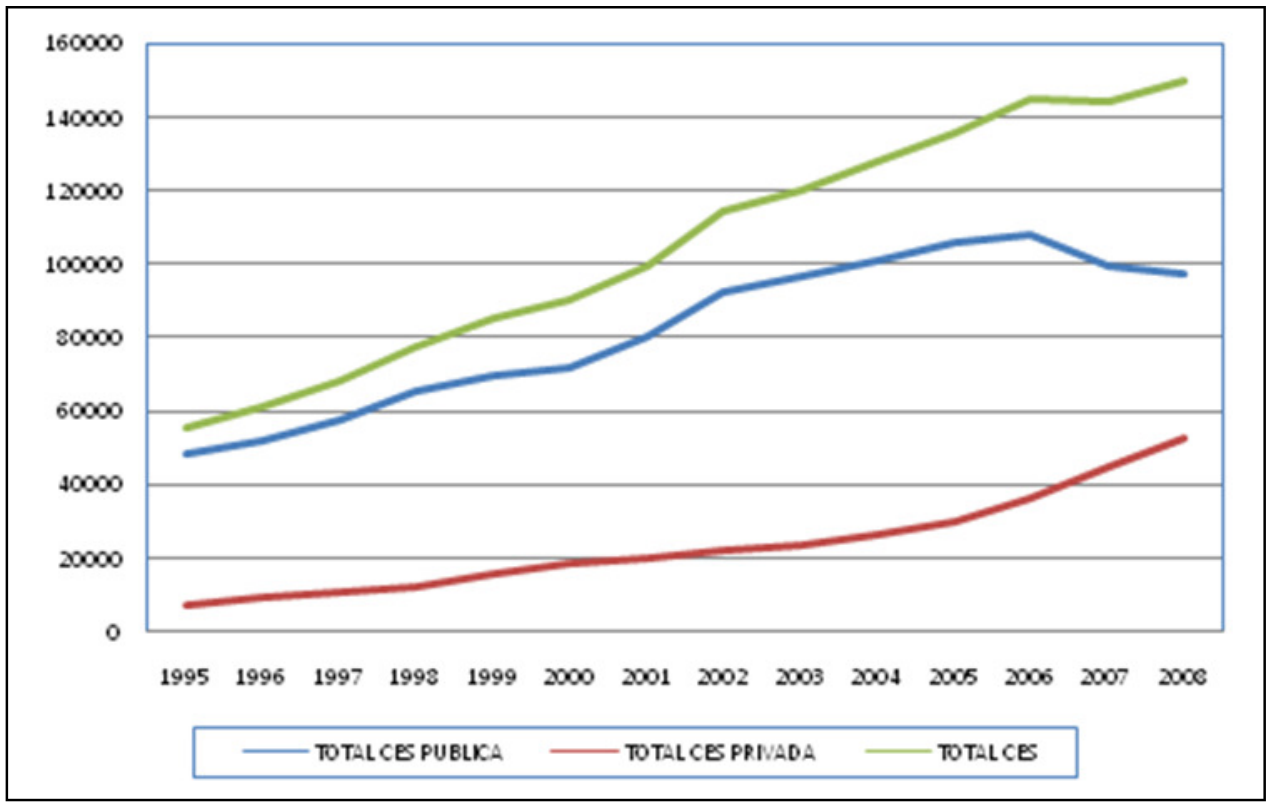

Gráfico 7, Honduras: matrícula total educación superior publica y privada, 1995-2008. (Fuente: Flores, M. 2011.) 
Por centro público y privado, la UNAH es la mayor contribuyente en estudiantes y supera abismalmente a los demás centros, la Universidad Pedagógica Nacional Francisco Morazán (UPNFM) que también es centro público se convierte en el segundo centro contribuyente de efectivos (Gráfico No. 8). En el caso de los demás centros, principalmente los privados contribuyen en volúmenes uniformes. En los últimos dos años la Universidad Tecnológica de Honduras (UTH) ha logrado ganar parte del mercado de la matrícula a través de una expansión de su cobertura geográfica. Cinco centros de veintiún absorben más del noventa por ciento del total de la matrícula, lo que muestra la alta concentración en pocos centros.

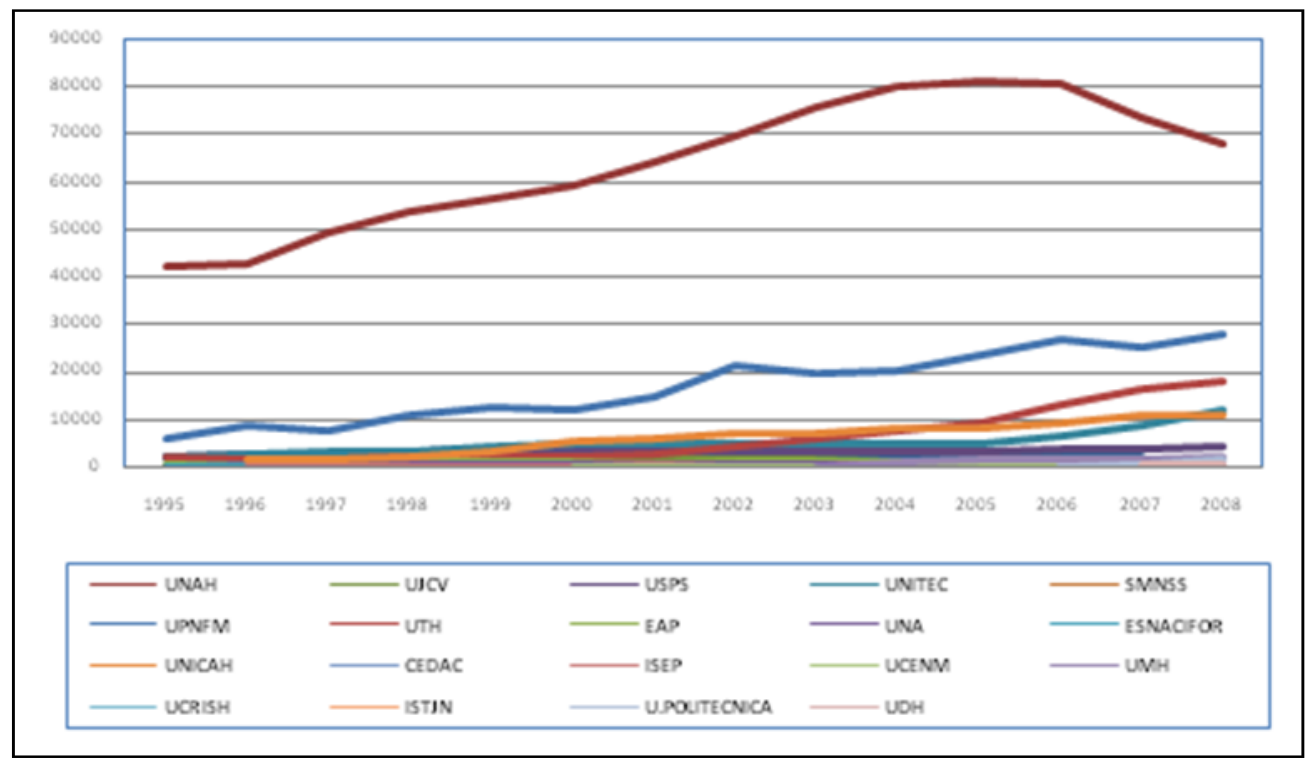

Gráfico 8, Honduras: matricula total en educación superior según CES, 1995-2008. (Fuente: Flores, M. 2011.)

\subsection{Matricula de primer ingreso}

Los centros de educación superior han aumentado el número de nuevos ingresos a través del tiempo (Gráfico No.9), en una década (1998-2008) su número aumentó de 17,646 a 27,933 estudiantes, aunque no fue de manera uniforme, ya que en el año 2002-2003 y el año 2007 se produjeron retrocesos en la tendencia, y se manifestaron en tasas de crecimiento interanual negativas, que en el último año superaron el 20\%. (DES, 2008) 


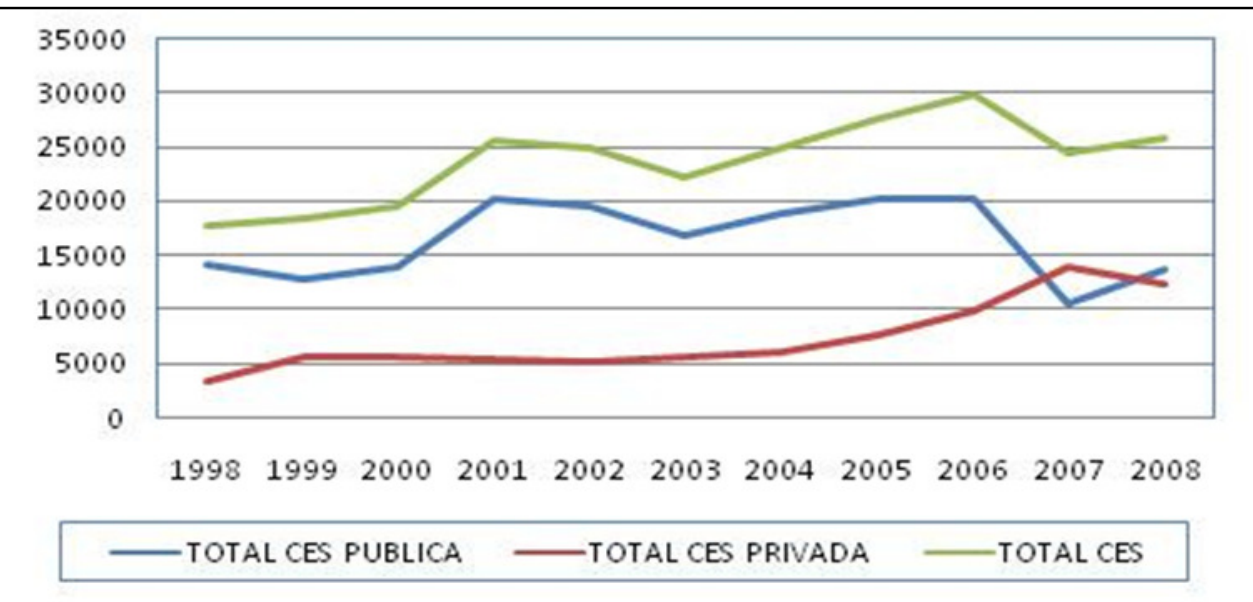

Gráfico 9, Honduras: matricula primer ingreso Educación Superior (publica y privada). (Fuente: Flores, M. 2011.)

Dichas reducciones en la tendencia en el flujo de efectivos de primer ingreso se debieron posiblemente por los picos producidos en la afluencia masiva en la matrícula de los años 2001-2002 en la UPNFM a los programas de formación docente, que incentivados por mejoras salariales en el estatuto del docente atrajeron ese flujo que consecuentemente terminado su afluencia en la matrícula de primer ingreso decayó (Gráfico No.10). A su vez en el año 2006 se implementa en la UNAH examen de admisión para estudiantes que les permitiría ingresar a estudiar el primer ingreso del 2007, tal examen provocó una disminución en la afluencia en la mayor universidad del país, lo que se reflejó en una tasa de crecimiento negativa del sesenta por ciento.

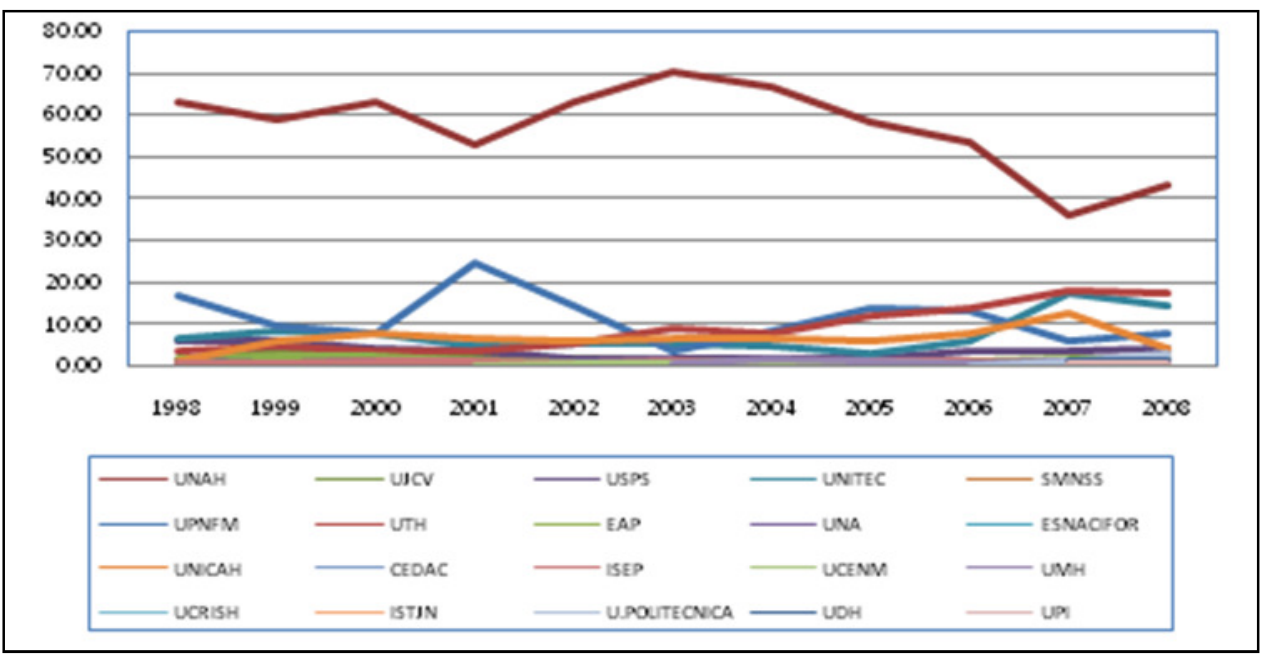

Gráfico 10, Honduras: Matrícula de primer ingreso en la Educación Superior, según CES (\%)

(Fuente: Flores, M. 2011.) 
La reducción en la matricula de los nuevos ingresos en la UNAH también se manifestó en la educación superior pública del país en comparación con la educación privada (Gráfico No. 11) que en los últimos diez años reduce su proporción del total, mientras en 1998 la matrícula de primer ingreso representaba el $81 \%$ del año 2008 su valor se ubicó en $52.5 \%$, dentro de este período en el 2007 se produjo por primera vez en la historia educativa superior del país que la matrícula de primer ingreso fuera más alta en los centros de educación privada.

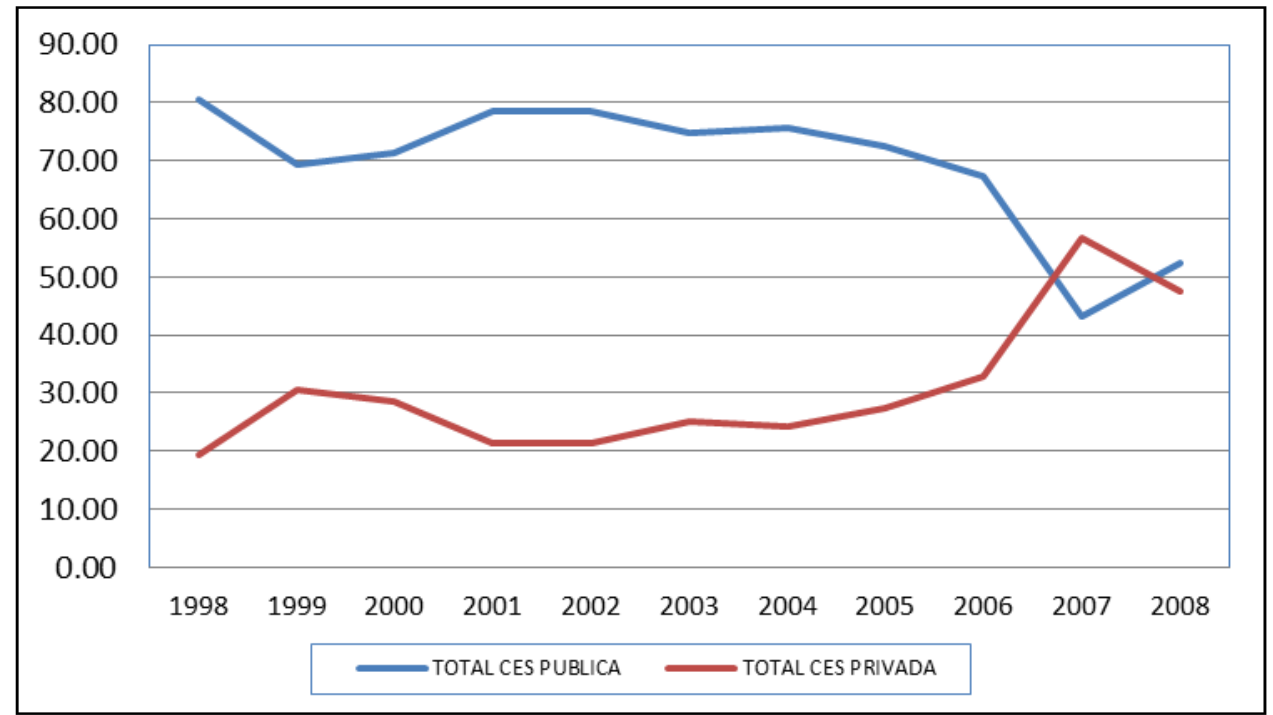

E\&A IIES

Gráfico 11, Honduras: matricula primer ingreso CES Publica y Privada, 1998-2008 (\%) (Fuente: Flores, M. 2011.)

\subsection{Resultados de graduación de las instituciones educación superior.}

A partir de los datos estadísticos recabados es plausible determinar que el número de egresados formales de la educación superior pública en Honduras se ha incrementado a través del tiempo. En los últimos diez años los graduados pasaron de aproximadamente 4,965 a cerca de 15,000 personas (Gráfico No.12), aunque los años 2001 y 2005 se produjeron cambios en la tendencia incremental, producidas posiblemente por los programas especiales de formación en ES brindados por la UPNFM, que graduaron estudiantes en los años anteriores. Asimismo, es notorio identificar que en el periodo antes descrito los graduados de la educación superior privada no sobrepasan el $50 \%$ de los graduados del sistema público, a excepción de los años 2006 al 2008 que logran alcanzar un número considerable. 


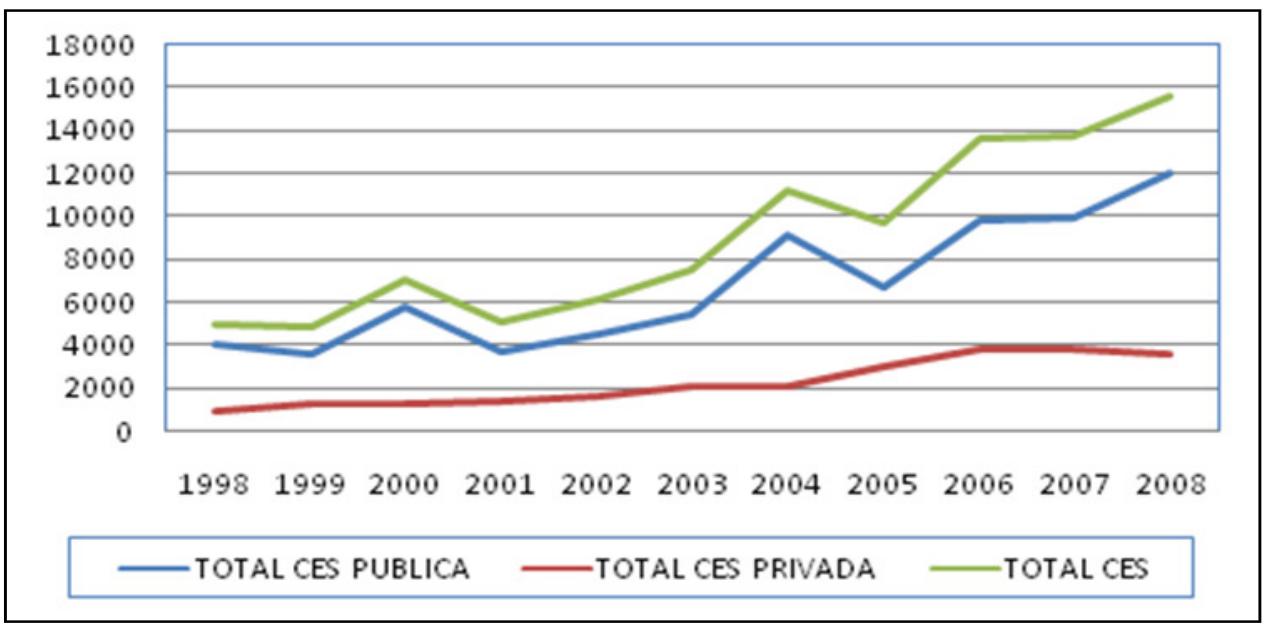

Gráfico 12, Honduras: graduados Educación Superior CES Publica y Privada,1998-2008 (Fuente: Flores, M. 2011.)

Vale la pena considerar que la educación superior pública es la que contribuye con un mayor número de graduados en la oferta laboral, solo las dos principales universidades públicas (UNAH y UPNFM) aportan con más de las dos terceras partes de los graduados universitarios en el país (Gráfico No. 13), diferenciándose la UPNFM, la cual logra insertar a sus egresados en al docencia, específicamente en el sector gubernamental.

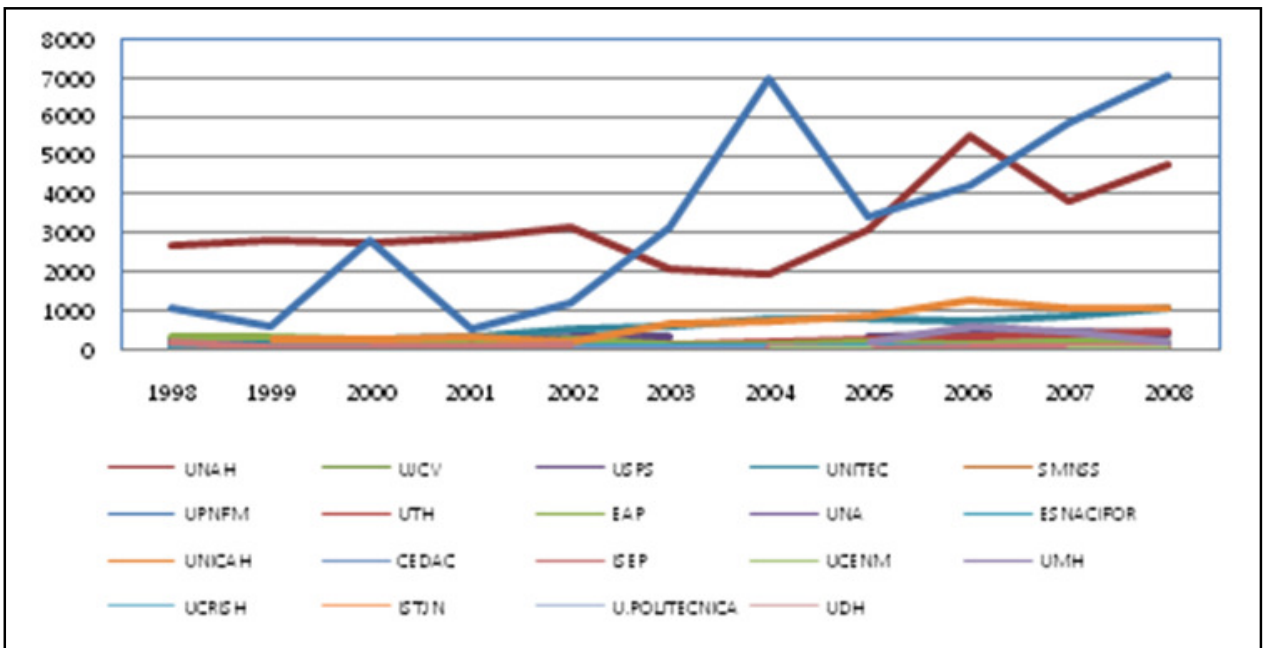

Gráfico 13, Honduras: graduados Educación Superior según CES, 1998-2008 (Fuente: Flores, M. 2011.) 
Con el transcurrir del tiempo, diversos estudios e instituciones, en función de las estadísticas correspondientes, han observado la feminización de la educación, que no es mas que un aumento de la matricula en el nivel de educación superior de las mujeres en proporciones similares o casi mayores a las de los hombres.

En el caso de los graduados en la última década es mayoritaria la cantidad de graduadas, incluso en el último año duplica su cantidad. En los centros de educación superior de mayor contribución en la formación de recursos humanos casi sin excepción se repite la tendencia de feminización de graduados (Gráfico No. 14).

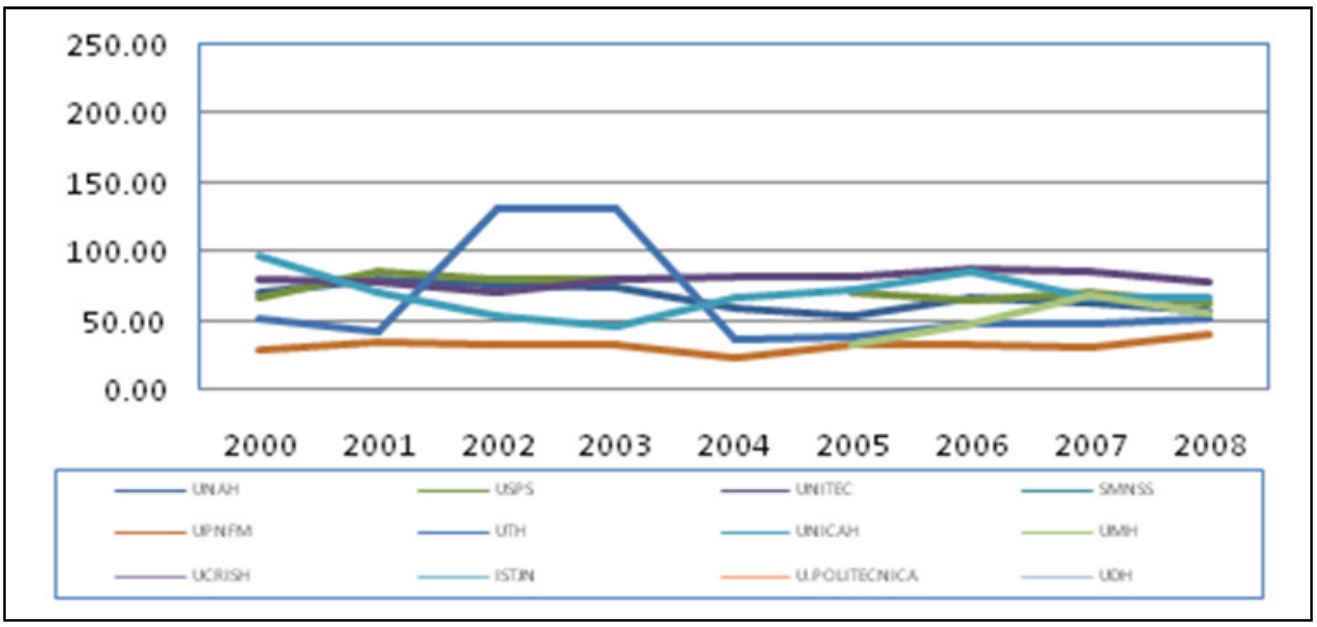

E\&A IIES

Gráfico 14, Honduras: Índice de masculinidad graduados en la Educación Superior 2000 -2008, según CES

(Fuente: Flores, M. 2011.)

\section{INSUMOS, PROCESOS Y RESULTADOS DE LA EDUCACIÓN SU- PERIOR}

Un resumen de la población universitaria hondureña indica que la matrícula total del sistema muestra una tendencia incremental a través del tiempo (Gráfico No. 15), lo mismo pasa con la cantidad de graduados cada año, sin embargo éstos, apenas han superado un décimo de la matrícula, tal situación indica que la inmensa mayoría se encuentra cursando estudios en dicho nivel. Teóricamente cada promoción estaría estudiando cinco años en los CES por lo que podría esperarse de acuerdo al flujo de la matrícula de primer ingreso que el número de estudiantes matriculados debería ser menor. 


\subsection{Coberturas educativas de educación superior}

En las décadas anteriores la cobertura de la educación superior en Honduras mostró niveles bajos, ya que solo habría cubierto entre el 11 al 15\% del total de población teóricamente apta para estar estudiando en ese nivel educativo, aunque es de observar que ha aumentado en las décadas recientes casi un tercio. (tabla 1). La cobertura educativa de la UNAH, principal centro educativo del país ha tenido un comportamiento contradictorio, primero tuvo incrementos hasta el año 2004 y de allí en adelante ha disminuido su tasa de cobertura (Tabla No. 1), similar situación se presenta en la educación superior pública a excepción que el punto de pico se produce en el año 2006. La cobertura de la educación privada tiene una tendencia alcista a través del tiempo, representando en el último año más de un tercio de la cobertura total de la educación superior. 


\section{Tabla No.1}

Honduras: Tasa de cobertura en la Educación Superior Publica y Privada, 2001-2008

\begin{tabular}{|r|r|r|r|r|r|r|r|r|r|}
\hline Año & $\begin{array}{c}\text { Población de } \\
18 \text { a 24 } \\
\text { años (INE) }\end{array}$ & $\begin{array}{c}\text { Matricula } \\
\text { total } \\
\text { UNAH }\end{array}$ & $\begin{array}{c}\text { Matricula } \\
\text { totales } \\
\text { (Público) }\end{array}$ & $\begin{array}{c}\text { Matricula } \\
\text { totales } \\
\text { (Privada) }\end{array}$ & $\begin{array}{c}\text { Matricula } \\
\text { totales } \\
\text { Honduras }\end{array}$ & $\begin{array}{c}\text { Cobertura } \\
\text { matricula } \\
\text { UNAH }\end{array}$ & $\begin{array}{c}\text { Cobertura } \\
\text { matricula es } \\
\text { público } \\
\text { HONDURAS }\end{array}$ & $\begin{array}{c}\text { Cobertura } \\
\text { matricula es } \\
\text { privada } \\
\text { HONDURAS }\end{array}$ & $\begin{array}{c}\text { Cobertura } \\
\text { matricula } \\
\text { totales }\end{array}$ \\
\hline 2001 & 914195 & 64142 & 79903 & 20025 & 99928 & 7.02 & 8.74 & 2.19 & 10.93 \\
\hline 2002 & 933784 & 69686 & 91994 & 22516 & 114510 & 7.46 & 9.85 & 2.41 & 12.26 \\
\hline 2003 & 951795 & 75643 & 96305 & 23572 & 119877 & 7.95 & 10.12 & 2.48 & 12.59 \\
\hline 2004 & 968592 & 80015 & 101222 & 26696 & 127918 & 8.26 & 10.45 & 2.76 & 13.21 \\
\hline 2005 & 984762 & 81099 & 105693 & 30139 & 135832 & 8.24 & 10.73 & 3.06 & 13.79 \\
\hline 2006 & 1001058 & 80476 & 108035 & 36757 & 144786 & 8.04 & 10.79 & 3.67 & 14.46 \\
\hline 2007 & 1017974 & 73212 & 99530 & 44974 & 144504 & 7.19 & 9.78 & 4.42 & 14.20 \\
\hline 2008 & 1036141 & 67666 & 97218 & 52810 & 150028 & 6.53 & 9.38 & 5.10 & 14.48 \\
\hline
\end{tabular}

Fuente: Flores, M, en base a datos de CNPV 2001 y Proyecciones de Población, INE.

\subsection{Oferta educativa reciente}

Si bien es cierto los Centros de Educación Superior (CES) deben responder a las dinámicas globales en cuanto a la adaptación de los programas académicos que ofrecen, éstos deben ir orientados a la satisfacción e inserción de los profesionales egresados de las CES a cumplir un rol de un mercado laboral y de una sociedad cada vez más competitiva. Sin embargo, dicha necesidad imperiosa no se ha visto e implementado con mucho ímpetu desde las CES de Honduras, ello debido particularmente a que se continúa ofreciendo ofertas académicas que pueden estar saturando el mercado de profesionales de las áreas curriculares que imparten, ello puede verse reflejado en el gráfico No.15

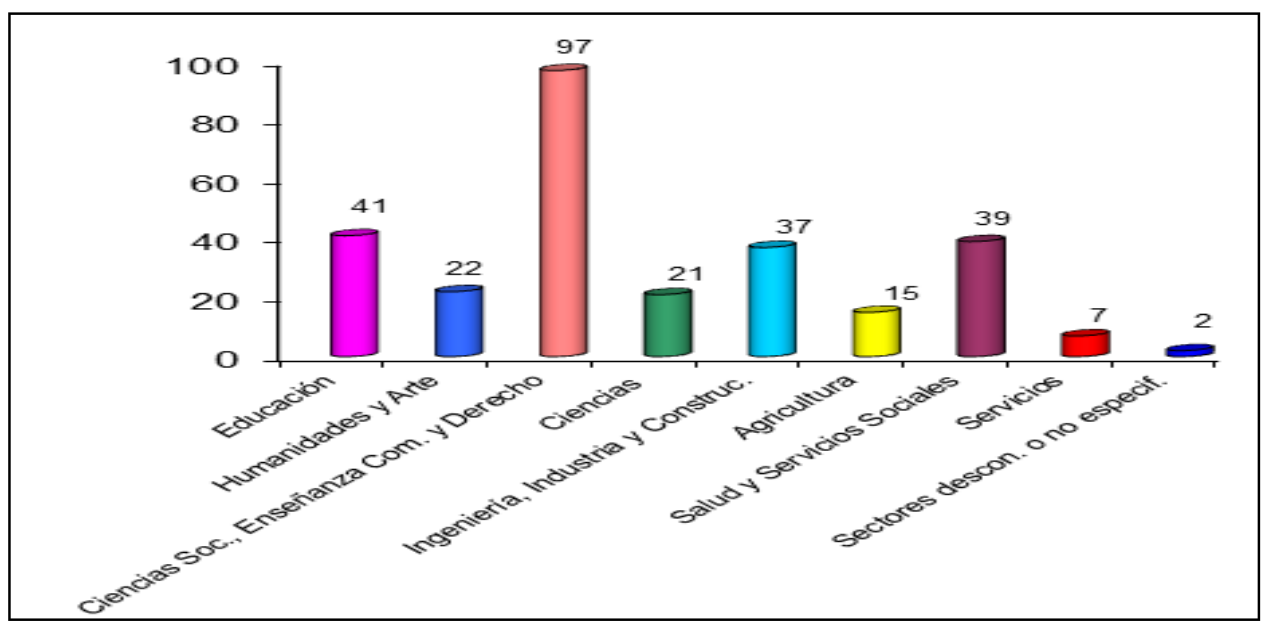

Gráfico 15, Oferta académica brindada en las CES Honduras, 2005 (\%)

(Fuente: Elaboración propia en base a Dirección de Educación Superior, anuario estadístico 2006.) 


\section{CONSIDERACIONES FINALES}

A partir de la descripción de los indicadores educativos previamente desarrollados, es plausible mencionar:

En Honduras durante el año 2010, el 15\% de las personas mayores de 15 años no disponían de la capacidad de leer y escribir. Además a nivel rural dicho indicador es presentado en una de cada cuatro personas que viven en esta zona de residencia.

Al considerar el analfabetismo por edades es importante mencionar que a partir de los 30 años este indicador aumenta, se vuelve preocupante debido a que en estas edades es donde aún se encuentra la población económicamente activa, que al presentar tales falencias les limita para tener una vida decorosa. A nivel nacional el indicador de analfabetismo es aun alto si consideramos que el estándar a nivel internacional para no sobrepasa el 5\%, mientras que honduras para el año 2010, dicho porcentaje llegaba a un $10 \%$.

Asimismo, se debe prestar atención al indicador de deserción escolar, ya que éste representa erogaciones adicionales de diversos sectores, es decir pues; la inversión realizada por los padres al mandar a los hijos a estudiar en el nivel correspondiente, así como la alta inversión que realiza el gobierno al asignar recursos adicionales por los jóvenes que abandonan el nivel y los que repiten dicho nivel o grado.

Debe realizarse esfuerzos conjuntos entre los diversos actores del sistema educativo (padres de familia, maestros, educandos, gobierno, asociaciones de cooperación, etc.) para permitir el desarrollo y la aplicación de políticas públicas orientadas a lograr mejores niveles de asistencia escolar en los niveles que muestran tasas sumamente bajas (diversificado, ciclo común).

A pesar de existir recientemente un auge en la expansión de las instituciones de Educación Superior en Honduras, aún persiste la prevalencia de la matrícula en las universidades públicas en relación a las privadas. A nivel de Oferta académica de las CES, se sigue manteniendo la tendencia a las carreras en el área de las ciencias comerciales y ciencias sociales, éstas prevalecen en relación a las carreras orientadas a las ciencias puras.

La cobertura educativa del nivel de educación superior para el periodo analizado se mantiene baja, es decir apenas llega al 15\% aproximadamente, si se compara tal indicador con países desarrollados o emergentes se observa que tal indicador muestra diferencias significativas. 
En la matricula total de educación superior se ha producido un cambio significativo en el volumen, aunque los centros de educación superior pública contribuyen con los mayores volúmenes, en los últimos dos años ha disminuido su número, básicamente por la reducción en la matrícula de la Universidad Nacional Autónoma de Honduras (UNAH), principal contribuyente de la educación superior en el país, que inclusive generó tasas de crecimiento interanual negativas y desde luego, por el incremento de matrícula en los centro de educación superior privada. En los últimos años la proporción de matriculados de educación superior privada se ha incrementado, de $13 \%$ a $35 \%$, la cual también es una tendencia general a nivel la región latinoamericana.

En los nuevos ingresos estudiantiles sobresalen las mujeres, en el 2008 el 56\% pertenecen al sexo femenino, e incluso instituciones como la UPNFM y la UNAH sus matrículas de primer ingreso son mayoritariamente femeninas, tendencia que viene de años atrás.

A nivel de egresados de nivel de educación superior se sigue manteniendo la tendencia de que egresan más profesionales de las instituciones públicas que de las instituciones privadas. 


\section{RECONOCIMIENTOS}

Esta investigación ha sido financiada parcialmente por el Consejo Nacional de Educación de Honduras, la Beca Sustantiva de Investigación No. 03-2011 por parte de la Dirección de Investigación Científica (DICU) y por la Vicerectoría académica de la UNAH. El autor desea agradecer el apoyo del Gobierno de Honduras y de la UNAH.

\section{REFERENCIAS}

Dirección de Educación Superior (2006). Anuario estadístico.

Flores, M. (2011), Oferta y demanda de educación superior: algunos elementos teórico-conceptuales y tendencias. IIES, UNAH.

Gobierno de Honduras, Constitución de la República de 1982 y sus reformas; Artículo 151.

INE (2010), Encuesta permanente de Hogares de Propósitos Múltiples.

INE (2001) Censo Nacional de Población y vivienda de honduras.

INE (2008) Encuesta Permanente de hogares de propósitos múltiples.

INE (2009) Encuesta Permanente de hogares de propósitos múltiples.

E\&A
INE (2010) Encuesta Permanente de hogares de propósitos múltiples.

Secretaría de Educación de Honduras (2009), Dirección de Planeamiento y Gestión, Informes Anuales 2000-2008.

UNAH, Dirección de Educación Superior (2008), Estadísticas de Educación Superior.

UNAH, 1985 Oferta y Demanda de Profesionales de Educación Superior en Honduras. 